EOMmun Communication et organisation

Or

La communication des nouvelles éthiques de

l'entreprise

\title{
Aperçus anthropologiques sur la question des normes sociales
}

Philippe Robert-Demontrond

\section{(2) OpenEdition}

\section{Journals}

Édition électronique

URL : http://journals.openedition.org/communicationorganisation/3271

DOI : 10.4000/communicationorganisation.3271

ISSN : 1775-3546

Éditeur

Presses universitaires de Bordeaux

Édition imprimée

Date de publication : 2 janvier 2005

Pagination : 44-55

ISSN : 1168-5549

Référence électronique

Philippe Robert-Demontrond, "Aperçus anthropologiques sur la question des normes sociales », Communication et organisation [En ligne], 26 | 2005, mis en ligne le 19 juin 2012, consulté le 01 ma 2019. URL : http://journals.openedition.org/communicationorganisation/3271 ; DOI : 10.4000/ communicationorganisation.3271

Ce document a été généré automatiquement le 1 mai 2019.

(c) Presses universitaires de Bordeaux 


\title{
Aperçus anthropologiques sur la question des normes sociales
}

\author{
Philippe Robert-Demontrond
}

1 Depuis peu sont développés, au plan mondial, des efforts de responsabilisation sociale des entreprises, de régulation des règles du jeu concurrentiel. Efforts de soutenabilité des systèmes de production visant à réduire leur contestabilité politique par la construction : 1) de clauses sociales, dispositions dans les ententes commerciales internationales permettant à un État de restreindre ses importations de biens produits dans des conditions violant des standards minimaux spécifiés dans ces ententes ; 2) de normes sociales, dispositions introduisant une discrimination positive supplémentaire, dans la mesure où elles peuvent se limiter à cibler les transnationales alors que les clauses sociales s'adressent, en théorie, à l'ensemble des entreprises d'un secteur économique national ; 3) de chartes sociales et de codes de conduite, finalement, dispositions librement adoptées par les entreprises et les engageant à respecter des standards minimaux du travail et d'organisation des conditions de travail - dans une perspective, notamment, de labellisation de leur production, de construction d'avantages concurrentiels.

2 Ces différents systèmes de régulation sociale de l'activité économique reposent sur de nombreux textes de lois et déclarations à portée universelle, servant de référentiels textes qui, visant l'uniformisation des règles du jeu concurrentiel, ont tous pour fondement principiel l'acceptabilité universelle des droits de l'homme. Ce qui, factuellement, n'est pas d'évidence. Or, la possibilité d'une relativité de ces droits, de l'existence d'une dimension culturelle, d'idiosyncrasies donc, a été négligée ou occultée lors de la rédaction de la Déclaration Universelle des Droits de l'Homme (DUDH), de 1948. Et ce, pour des raisons idéologiques mais aussi pratiques - peu d'États autres qu'occidentaux ayant effectivement participé à leur élaboration. De là finalement, passant sous silence toute autre conception, une excessive influence de la tradition occidentale des droits de l'homme sur la rédaction de la DUDH - de sorte que les normes qu'énonce celle-ci ne reflètent pas toujours celles de certaines autres traditions 
axiologiques, philosophiques ou religieuses, quand elles ne reposent pas sur des traits axiologiques en opposition ou en contradiction.

Il s'agit ici de montrer que la mondialisation n'a jusqu'à présent aucunement correspondu à une uniformisation des comportements et des mentalités. De façon plus nuancée, elle s'est traduite à la fois par la progression de certaines tendances à l'homogénéisation et par un mouvement inverse de résistance sous la forme d'une résurgence des particularismes culturels et des revendications identitaires.

\section{Non pas unité, mais diversité des référentiels axiologiques}

4 Notre examen des référentiels axiologiques s'inscrit ici dans le prolongement des travaux d'Alliot (1989) qui, «s'il y a un trait commun entre toutes les sociétés, c'est bien que chacune construit son propre univers mental, porteur de modèles fondamentaux et dispensateur de sens, que révèlent à la fois la vision du monde visible et invisible de chacun de ses membres (...) Qui veut comprendre la forme et les institutions juridiques d'une société a donc intérêt à les rapporter non aux institutions de sa propre société - le rapprochement serait superficiel - mais à l'univers de celle dans laquelle il les observe ».

Dans cette perspective, deux formes civilisationnelles sont analysées, en examinant respectivement: 1) leurs archétypes; 2) l'incidence de ces archétypes sur leurs conceptions du droit ; 3) l'incidence de ces archétypes sur leurs conceptions des droits de l'homme. Ces deux formes civilisationnelles retenues sont celles assises sur l'hindouisme et sur l'animisme. Le choix résulte essentiellement, pour la première, de ce qu'elle coïncide avec une puissance géopolitique de première importance, tant en termes démographiques qu'économiques, avec qui plus est une dynamique assurant de considérables perspectives de croissance et un élargissement continu, sur le siècle, du potentiel d'influence. Pour la seconde forme civilisationnelle, le choix résulte de ce qu'elle correspond à un système culturel actuellement partagé par des centaines de millions d'hommes, que l'on ne peut oublier sous prétexte de leur pauvreté - du caractère marginal de leur contribution à l'économie mondiale.

\section{Les droits de l'homme au regard de l'hindouisme}

6 L'hindouisme est tout entièrement fondé sur le concept, issu de la tradition védique, de dharma - terme polysémique renvoyant, par sa structure étymologique même, à un principe de cohésion cosmique, comme «ce qui soutient». Le dharma est, fondamentalement, ce qui préserve du chaos, ce qui tient, ce qui maintient le monde, et le dharma lui-même se maintient par des rites. De là donc son acception, également, comme l'ensemble des valeurs éthiques. Et de là le fait que l'hindouisme définit un cadre civilisationnel qui, par essence, "n'a rien à faire de la mise en évidence du "droit" d'un individu contre un autre ou de l'individu vis-à-vis de la société, mais se préoccupe plutôt d'établir le caractère dharmique (juste, vrai, consistant, ...) ou adharmique d'une chose ou d'une action » (Panikkar, 1984). Quant à l'action, justement, l'homme n'est pas conçu, dans l'hindouisme, comme un être de besoins et de désirs à assouvir, mais comme un être ayant pour obligation de se libérer de ses besoins, d'éteindre ses désirs. L'idéal de l'existence n'est alors pas d'apprendre à accumuler des richesses, mais à savoir s'en 
départir - d'où l'accent donné dans toute la formation hindoue à l'aparigraha: la nonpossession (Vachon, 2000), Ainsi donc, si le respect des devoirs du dharma, la poursuite des profits matériels (artha) et du plaisir sensuel, l'accomplissement du désir (kama), sont les buts premiers (purushârtha) de l'homme, selon la tradition védique, la quête de la délivrance (moksha) leur est supérieure, car elle nie leurs finalités mondaines.

Droit et dharma présupposent des philosophies, reposent sur des conceptions du monde et de la place de l'homme dans le monde dont les différences sont significatives, et ne sont pas superficielles (Alam, 1999). L'homme lui-même n'est jamais, dans la perspective de l'hindouisme, "qu'un noeud formant partie intrinsèque du réseau de relations qui constitue l'étoffe du réel » (Panikkar, 1984). Chaque existence est un réseau de relations «dans la réciprocité, la mutualité, la communauté»(Kumar, 2000). Relations qui inscrivent constamment l'individu en situation de débiteur, devant gratitude : 1) aux sages (rishi-rina) ; 2) aux ancêtres (pitry-rina) ; 3) aux divinités et forces de la nature (divarina) - ainsi est constitué le sva-dharma, c'est-à-dire, le dharma propre à la situation humaine (Vachon, 2000). Plus encore, dans le prolongement immédiat de la religion védique, la continuité et la prospérité du monde sont figurées dans l'hindouisme reposer sur un processus sacrificiel - dont la principale victime est l'homme. Le mythe du sacrifice de l'homme cosmique (Purusha), définissant la société comme un tout organique, est ainsi essentiel qui : 1) énonce les principes d'une division sociale du travail ;2) insiste sur l'aspect profondément relationnel de l'existence ; 3) prononce la primauté de l'essence sur existence. Le dharma apparaît en conséquence comme un système de "devoirs » n'ayant cependant rien d'une obligation exogène, et ne requérant aucunement quelque espèce de pression ou de coercition, parce que s'agissant, fondamentalement, de l'ordre naturel des choses.

8 En conséquence de cet archétype culturel, vient l'insistance sur : 1) l'idée que « les droits de l'homme ne sont pas seulement des droits de l'homme individuels »;2) l'idée que « les droits de l'homme ne sont pas des droits de l'homme seulement " - les créatures animées comme celles supposées inanimées étant aussi impliquées dans le dharma ; 3) l'idée que « les droits de l'homme ne sont pas seulement des droits »- mais sont aussi des devoirs, ces deux aspects étant interdépendants. C'est l'harmonie du cosmos qui prime (Panikkar, 1984).

\section{Les droits de l'homme au regard de l'animisme}

Dans la perspective de la pensée animiste, dominante en Afrique, l'univers est doué d'un principe vital, l'anima, régulé par le mouvement, la circulation continue d'énergies, qui cherchent à se compléter mutuellement, harmonieusement. Et ce, en termes d'autoorganisation : il n'y a pas, dans les cosmogonies des sociétés animistes, de Dieu créateur, extérieur à sa création. Le monde émerge progressivement du chaos qui, indistinct, contenait déjà tout l'avenir en puissance. Le monde se crée et se maintient à chaque moment et l'homme y joue un rôle primordial, pour aider à préserver l'harmonie de l'univers. Dans cette vision du monde, ce n'est pas un ordre imposé, extérieur et uniforme qui est à l'origine de l'unité de la société, mais au contraire l'affirmation de groupes divers, fondamentalement complémentaires et solidaires. La cohérence de la société, son unité, ne passe pas par l'uniformité, au contraire. La différence est conçue comme le fondement de l'unité, les législations uniformisantes, comme les législations occidentales, sont perçues comme destructrices de l'unité. De plus ce rejet de lois supérieures 
immuables auxquels ils pourraient se soumettre, rend les hommes responsables eux même de leur propre futur et mène à une valorisation de la conciliation et d'un esprit unanimiste (Alliot, 1983, 1989). La coutume aussi reflète cet idéal sociétal, car loin d'être un recueil de normes intangibles, comme on l'a longtemps conçue, elle correspond bien plutôt à une manière ancestrale de faire, interpellée pour trouver des solutions à des situations particulières (Le Roy, 1995; Marie, 1997). Elle est donc moins norme ou procédure que processus, et est conforme à l'idéal du règlement des conflits en interne, plutôt que par le recours à une instance extérieure.

10 Ce cadre conceptuel exerce une considérable influence sur le droit et la pensée du politique, avec: 1 ) des économies plus axées sur la redistribution (prévalence de l'espace public) que sur l'acquisition (prévalence de l'espace privé) : 2) des politiques s'enracinant plus dans le consensus, dans la palabre (prévalence de l'espace public), que dans l'opposition conflictuelle des idées (prévalence de l'espace privé). De même que dans l'hindouisme, l'essence l'emporte sur l'existence - de sorte encore que, contrairement à l'Occident où l'individu conserve, sa vie durant, un statut juridique identique, des droits invariables, et où tout individu se voit reconnu des droits identiques, le statut individuel, le régime de droits individuels, est déterminé par les fonctions exercées.

11 Cet archétype culturel a évidemment de très fortes incidences sur l'acception des droits de l'homme, qui se traduisent pas une insistance toute particulière sur :1) une notion de devoirs, liés à l'appartenance individuelle à un groupe social ; 2) une définition de la dignité individuelle par le statut social, à assumer, par le rôle social, à accomplir ; 3) une conception de la revendication de droits individuels telle que dévalorisant la dignité liée à l'appartenance sociale; iv) une conception de la primauté du groupe social telle qu'inclinant à accorder la priorité à la préservation du tissu social, et à une obligation de solidarité à l'égard du groupe social (Mbaye, 1992). La Charte de Banjul, adoptée par l'Organisation de l'Unité Africaine en 1981, reprend les principes de la DUDH pour ce qui est des libertés fondamentales, reconnues sans aucune dérogation, et pour ce qui est des droits économiques, sociaux et culturels, mais pose également des principes originaux : 1) en donnant une place importante au droit des peuples, inscrivant ce principe, nouveau, dans l'intitulé même de la Charte ; 2) en équilibrant les droits de l'homme par des devoirs, par une valorisation du concept de personne (dont la dimension sociale est essentielle) face au concept d'individu ; 3) en insistant sur l'obligation de veiller « à la préservation et au renforcement des valeurs culturelles africaines positives, dans un esprit de tolérance, de dialogue et de concertation et d'une façon générale de contribuer à la promotion de la santé morale de la société " (art.29), affirmant ainsi l'existence d'un devoir à la différence, tant que celle-ci est positive - et non pas uniquement d'un droit à la différence. Le fait que soit également préconisé de résoudre les litiges par la conciliation est typique de l'archétype culturel animiste.

\section{Non pas convergence, mais divergence des référentiels axiologiques}

12 Au total, il est dans les faits une diversité des systèmes axiologiques dont les traits caractéristiques, les particularismes s'opposent, virtuellement, à l'universalité des droits de l'homme: ainsi de l'hindouisme et de l'animisme, comme on l'a vu, mais aussi du bouddhisme (Dagpo-Rimpoche, 1990) et du confucianisme (De Bary, 1998; Li, 1998; Rouland, 1998) ou encore de l'islam (Aldeeb Abu-Sahlieh, 1994). 
13 L'actualisation même de cette opposition est quant à elle fonction de l'affirmation, ou non, d'une relativité des traditions culturelles: autrement dit, est fonction du développement d'un discours: 1 ) déniant toute espèce de supériorité d'un système culturel sur un autre; 2) affirmant a contrario l'incommensurabilité des systèmes culturels. Or, ce type de discours, usuellement dit "post-moderne", est actuellement tenu et s'impose de plus en plus en rupture avec celui originel, moderne, au fondement des déclarations politiques prétendant à l'universalité de facto et à l'universalisation de jure des droits de l'homme.

Reste que, nombre de théoriciens notent la convergence en cours des référentiels axiologiques. Le relativisme serait alors une erreur d'intellectuels, une aberration conceptuelle - la diversité étant temporaire, du fait d'une absence effective de relativité, de la supériorité effective d'une culture sur les autres. «Il est possible, sur le plan de la logique abstraite, que chaque culture soit incapable de porter un jugement vrai sur une autre culture puisqu'une culture ne peut s'évader d'elle-même et que son appréciation reste, par conséquent, prisonnière d'un relativisme sans appel. Mais si l'on regarde autour de nous, depuis un siècle, loin de rester enfermées sur elles-mêmes, toutes les civilisations reconnaissent, l'une après l'autre, la supériorité de l'une d'entre elles, qui est la civilisation occidentale». Cette affirmation de Lévi-Strauss (1952), qui parut notablement confortée sinon confirmée par la chute du Mur - avec alors même l'idée selon laquelle, finalement, l'on n'aurait plus qu' « un seul et même monde » (Fukuyama, 1992) - est factuellement contredite par l'évolution dernière de celui-ci.

mondialisation, souvent perçu comme étant d'uniformisation culturelle, de convergence donc, est de fait plus précisément vécu, la plupart du temps, comme une " occidentalisation du monde " (Latouche, 1992) sinon comme une " américanisation du monde » - agressive, privative d'identité, dépossessive des repères existentiels. Et les résistances à ce processus que l'on voit alors émerger ne sont pas seulement positives, tenant en l'affirmation d'une originalité, d'une spécificité fortement revendiquée, mais sont également négatives, consistant alors en un refus, éventuellement violent, du mouvement de globalisation - pour la défense des particularismes nationaux, régionaux, locaux, contre toute altération des référentiels axiologiques « traditionnels ».

Ces résistances s'opposent, en première instance, au mouvement de marchandisation du monde et correspondent, sur le fond, à la cristallisation d'affects nostalgiques. Au plan psychologique, ceux-ci peuvent être appréhendés comme des mécanismes adaptatifs, ayant pour vertu d'aider les individus à s'ajuster aux changements, à maintenir leur identité face aux transitions et perturbations majeures les affectant (Robert-Demontrond, 2002). Les affects nostalgiques contribuent de fait à compenser les difficultés psychologiques liées à ces transitions, et s'apparentent en conséquence à des réactions défensives contre le changement, visant à l'affaiblissement des blessures affectives, narcissiques, que celui-ci provoque. Prévaut alors l'impression d'une restauration de soi, d'une affirmation de l'ipséité individuelle contre les changements ou les altérations du monde. Et il en va très exactement du corps social comme des individus qui le constituent et l'instituent: chaque société assure son identité dans l'intégration de son passé, de la même façon exactement que la conscience de soi-même est conscience de son passé. De là l'engagement, actuellement, au plan mondial, d'un processus sociétal de « réarmement identitaire » (Robert-Demontrond, 2002), de «revivalisme culturel» (Badie \& Smouts, 1999), processus de «re-tribalisation culturelle» (Premdas, 1997) allant à l'encontre d'une modernité vécue très souvent comme génératrice d'exclusions, de frustrations, 
d'insécurité économique et ontologique, allant à l'encontre de la mondialisation - dans ce qu'elle représente de perte de soi ou de risque de perte de soi. Avec donc en final une crispation sur les référentiels axiologiques traditionnels - notamment ceux civilisationnels, entendus ici comme ceux philosophiques et religieux.

Surtout : ces résistances émotionnelles à l'universalisation des références axiologiques sont d'autant plus vives qu'elles ont également pour fondement l'observation d'une actuelle instrumentalisation stratégique de la DUDH, à des fins économiques. Il convient, pour mieux pénétrer ce point, de rappeler que la stratégie se réfléchit à trois niveaux : 1) la symétrie, qui correspond à une logique de confrontation selon des modalités opérationnelles identiques; 2) la dissymétrie, qui résulte de la recherche d'une supériorité quantitative et/ou qualitative dans les modalités opérationnelles;3) l'asymétrie, qui résulte de la recherche d'une supériorité opérationnelle par l'exploitation des faiblesses de l'adversaire, tout en évitant ses points forts élargie à l'ensemble du champ social de la conflictualité, l'asymétrie correspond à l'utilisation d'une « règle du jeu » différente, l'introduction de nouvelles modalités opérationnelles, permettant d'acquérir l'avantage sur l'adversaire en le déstabilisant. Ce comment, justement, l'universalisme des droits de l'homme est de plus en plus perçu.

Sur ce plan, quelque espèce de "sentiment d'une supériorité illusoire ", déjà dénoncé par Soljénitsyne (1978, p.12), entretiendrait l'idée que tous les pays doivent suivre, absolument, le mode de développement, le chemin de croissance ouvert par les systèmes occidentaux - « théoriquement les meilleurs » (Soljénitsyne, 1978, p. 13).

On ne peut négliger, dans l'apparent suivi universel de ce chemin, le fait que le discours des bailleurs de fonds internationaux - conçu " pour conditionner de manière uniforme tous les pays par rapport à un unique référent éludant la prise en compte des spécificités historiques et culturelles» (Boukamani, 2000) - a pour effets : 1) des ralliements (la ratification des textes internationaux sur les droits de l'homme) effectués par calcul et non par conviction ; 2) une dénonciation récurrente de néo-colonialisme, économique et culturel (une perception de la rédaction des clauses sociales, normes sociales, chartes sociales, comme relevant d'un protectionnisme masqué). Nombre des États qui, récemment encore, étaient des colonies, ont ainsi tendance à affirmer avec insistance que la question du respect des droits de l'homme doit être compatible avec la notion de souveraineté (Badie, 1999) et sont poussés à dénoncer comme "impérialisme de la vertu », selon l'expression de Dezalay et Garth (2000), l'effort de responsabilisation sociale des entreprises et de régulation sociale des règles du jeu concurrentiel.

Au total, et la résistance émotionnelle à la "main invisible des marchés ", au processus d'uniformisation des modes de vie par la mondialisation des politiques et pratiques commerciales, et la résistance émotionnelle à la "main visible des politiques ", à l'exploitation cynique du droit international pour la construction de barrières nontarifaires, sont au fondement d'un rejet de la déclaration universelle des droits de l'homme. Rejet qui n'est pas conceptuellement infondé : il y a bien une étrangeté de ces droits, une altérité radicale aux imaginaires de populations en nombre. Etrangeté, altérité, qui ne peut être plus longtemps ignorée, ou minorée. 


\section{Conclusion : pour une construction dialogique des référentiels normatifs}

21 Depuis près d'un siècle à présent, praticiens et théoriciens du management ont négligé de considérer l'étendue de la dépendance aux systèmes de valeurs de leurs objets. Ceci, notamment $d u$ fait du voile jeté sur ce rapport fonctionnel par l'existence d'une relative homogénéité culturelle entre les acteurs économiques - de leur appartenance au même champ civilisationnel. L'apparition des pays d'Asie dans le jeu économique, l'irruption d'acteurs intellectuellement, philosophiquement différents de l'Occident, a introduit une rupture de règles du jeu du même ordre que celle apparue, en son temps, avec l'apparition du protestantisme.

Ce qui permit à ces pays d'Asie d'être concurrentiels, supérieurement compétitifs c'est, fondamentalement, leurs rapports à l'action, leurs conceptions de la place de l'homme dans les organisations sociales, de ses droits, de ses devoirs. La compétition économique, en d'autres termes, ne se déroule pas seulement entre pays de niveaux de vie différents, mais également, et surtout, entre pays de modes de vie, de styles de vie différents, ne partageant pas les mêmes conceptions du monde, les mêmes représentations mythiques, archétypales, de l'homme, de la société, etc. Ce qui, de ce fait, signifie qu'ils ne respectent pas les mêmes règles du jeu.

Le développement des politiques de responsabilité sociale des entreprises s'interprète ainsi, fondamentalement, comme un effort d'harmonisation de ces règles du jeu. Explicitement: les clauses sociales, dans les relations internationales, visent à la réduction des distorsions de concurrence qu'induisent les écarts de traitement des salariés. Implicitement : en se souciant des droits de l'homme, de leur respect dans les systèmes de production, en s'assignant comme mission leur promotion universelle, les tenants du commerce équitable et du commerce éthique œuvrent pour l'uniformisation des règles du jeu - pour l'imposition des règles du jeu de l'Occident: son rapport à l'action, au travail, sa conception de la place de l'homme dans les organisation sociales, de ses droits, de ses devoirs.

Il faut être éthique, il faut respecter les droits de l'homme : lesquels? Destinée à régler définitivement la question, la position officielle de l'ONU, transcrite en 1993 par la Déclaration de Vienne - qui affirme en son premier paragraphe que le caractère universel des droits de l'homme est «incontestable »-, masque très abusivement la réalité du problème. Il paraitt nécessaire, au contraire de l'adoption d'une telle position de principe, factuellement contredite, de prendre acte de ce que les référentiels axiologiques (et cosmologiques, ontologiques) ne sont pas unanimement partagés, et de ce que cela n'est pas sans incidence quant à la façon dont est pensé, et dont est pensable, la soutenabilité du développement économique. 


\section{BIBLIOGRAPHIE}

Alam J. (1999), India. Living with modernity, Oxford University Press.

Aldeeb Abu-Sahlieh, Sami A. (1994), Les musulmans face aux droits de l'homme, Bochum, Winkler.

Alliot M. (1983). « Anthropologie et juristique ». 1953-1989 Recueil d'articles, contributions à des colloques, textes du Recteur Michel Alliot, Paris, LAJP, 207-241.

Alliot M. (1989). « La coutume dans les droits originellement africains ». 1953-1989 Recueil d'articles, contributions à des colloques, textes du Recteur Michel Alliot, Paris, LAJP, 269-290.

Badie B. (1999), Un monde sans souveraineté, Paris, Fayard.

Badie B., Smouts M-C. (1999), Le retournement du monde, Paris, Dalloz.

Boukamani B. (2000), « La "bonne gouvernance" et l'État en Afrique », Bulletin de Liaison, 25, 61-80, ww.dhdi.free.fr/recherches/bulletins/bull25.pdfs.

Dagpo-Rimpoche (1990), «Les Droits de l'Homme vus par un Bouddhiste », in A. Lapeyre, F. de Tinguy, K. Vasak (eds.), Les dimensions universelles des Droits de l'Homme. I. Les dimensions spirituelles et intellectuelles des Droits de l'Homme, Bruxelles, Bruylant, 175-188.

De Bary W. M. T. (1998), Asian values and Human Rights. A Confucian Communitarian Perspective, Harvard University Press.

DeGeorge R. (1993), Competing with Integrity in International Business, Oxford University Press. Dezalay Y., Garth B. (2000), « L'impérialisme de la vertu », Le Monde diplomatique, mai, 8-9.

Donaldson T. (1989), The Ethics of International Business, Oxford University Press.

Donaldson T., Dunfee T. (1999), "When Ethics Travel: The Promise and Perils of Global Business Ethic"s, California Management Review, 41, 4, 45-63.

Kumar S. (2000). "You are, therefore I am". Resurgence, 19, 7.

Le Roy É. (1992), « Un droit peut en cacher un autre », Informations sociales, 22, 10-19.

Le Roy É. (1995). « L'accès à l'universalisme par le dialogue interculturel ». Revue Générale du Droit, 26, 5-26.

Li X. (1998), «Les droits de l'homme en Chine. Réalité et polémique », Droit et Cultures, 35, 107-126.

Mbaye K. (1992). Les droits de l'Homme en Afrique. Pédone.

Marie A. (1997), L'Afrique des individus. Paris, Khartala.

Panikkar R. (1984). « La notion des droits de l'homme est-elle un concept occidental ?». Interculture, 27, 1, 3-27.

Fukuyama F. (1992), La fin de l'histoire et le dernier homme, Paris, Flammarion.

Latouche S. (1992), L'occidentalisation du monde, Paris, La Découverte.

Robert-Demontrond P. (2002), « La nostalgie : du refus de l'altérité à la quête de l'ispséité », Arobase, Journal des lettres et sciences humaines, 16, 1, 19-29. 
Robert-Demontrond P. (2005), « En aperçu sur les enjeux et limites de l'évaluation contingente : la mesure du consentement à payer pour une labellisation sociale de l'offre commerciale », in M. Legall-Ely \& P. Robert-Demontrond (eds), Méthodes d'évaluation contingente et d'analyse conjointe, Rennes, Apogee.

Rouland N. (1998), «La doctrine juridique chinoise et les droits de l'homme », Revue Universelle des Droits de l'Homme, 10, 1-2, 1-26.

Soljénitsyne S. (1978), Le déclin du courage, Paris, Le Seuil.

Sudhir Kakar M. (1985). Le monde intérieur. Paris, Les Belles Lettres.

Vachon R. (2000). Au-delà de l'universalisation et de l'interculturisation des droits de l'homme, du droit et de l'ordre négocié. Bulletin de liaison, 25, septembre, 9-22.

\section{RÉSUMÉS}

Les stratégies managériales en "responsabilité sociale " sont de plus en plus souvent fondées sur des textes de portée universelle, comme la Déclaration Universelle des Droits de l'Homme, adoptée par les Nations Unies en 1948. Avec alors comme présupposé l'idée que cette Déclaration promeut des valeurs effectivement universelles. Ce que contredit cet article, à partir d'une mythanalyse des religions hindouistes et animistes.

Usually grounded on Universal Declarations, and especially on the "Universal Declaration of Human Rights" adopted by the United Nations in 1948, managerial strategies of "corporate social responsibility" presuppose that this declaration promotes universal values. This paper aims a criticism to this occidental "evidence". A mythanalysis of hindhuist and animist religions shows that they are based on values that we do not find in the "presupposed" universal declaration. This conflict of values involves that with its current contents, this declaration cannot be applied everywhere.

\section{AUTEUR}

\section{PHILIPPE ROBERT-DEMONTROND}

Philippe Robert-Demontrond est professeur des Universités à Rennes, directeur de l'équipe de recherche en marketing-management du CREM, Centre de Recherche en Economie et Management, UMR CNRS et directeur de l'IREIMAR, Institut de Recherche Européen sur les Institutions et les MARchés, fédération de recherche CNRS. Mail : philippe.robert@univ-rennes1.fr 